standardised incidence ratio (SIR) by comparing with the general population. Subgroup analyses were performed based on the types of immune-mediated diseases and biologic drugs.

Results A total of 2485 patients with immune-mediated diseases were identified $(10.6 \%$ IBD, $77.7 \%$ rheumatology, and 11.8\% dermatology). 54 subjects developed active TB during 6921 person-years of follow-up. The mean age was 43 years, and the median follow up duration was 748 days. The overall SIR for TB in patients with included immune-mediated disease was 10.91. Patients with IBD had an over 17-fold increased risk of TB compared to the general population (SIR, 17.53), and patients on infliximab had a nearly 26 -fold increased risk of TB compared to the general population (SIR, 25.95). Risk of TB was highest in the age group 50-59 years (SIR, 15.72). The risk of $\mathrm{TB}$ with infliximab was higher than etanercept (hazard ratio $[\mathrm{HR}], 4.10$ ) and adalimumab (HR 2.08). No significant difference in the risk of TB was observed among the subgroups of IBD, rheumatology and dermatology.

Conclusions In the population-based study, biological therapy is associated with higher risk of TB in patients with immunemediated diseases compared with the general population, and infliximab is associated with the highest risk of TB amongst all biologics. This study suggests patient and biologic selection may be important in balancing the benefit and risk of TB when treating patients with immune-mediated diseases.

\section{IDDF2018-ABS-0062 FUNCTIONAL OUTCOME OF HIRCHSPRUNG'S DISEASE, POSTSURGICAL TRANSANAL ENODORECTAL PULLTHROUGH AT NATIONAL CHILDREN'S HOSPITAL}

Meliza Ylaine Salvador*. Clinical Chief Resident of National Children's Hospital, Philippines

\subsection{6/gutjnl-2018-IDDFabstracts.94}

Background Hirschprung's Disease is a congenital illness secondary to lack of ganglion cells in the intestinal tract leading to mechanical obstruction. In the Philippines, Hirschsprung's Disease ranks 9th over the top 10 cases causing morbidity to Filipino children and the mortality rate can reach up to $50 \%$. The treatment is still surgery, such as Soave procedure.

Design Descriptive cross-sectional study.

Subjects The participants of the study were followed up patients at the Surgery Out Patient Department from March 2016 to August 2017, ages 0-7 years old, diagnosed with Hirschsprung's Disease, post Transanal endorectal pull-through. Methodology Purposive sampling was used to select participants. The sample size was 40 based on the proportion of good functional outcome among patients who underwent transanal endorectal pull through.

Statistical Analysis Descriptive Analysis using proportion and percentages were used to present the results in all the variables.

Results Out of the 40, $82 \%$ were diagnosed with the disease as early as the newborn period, and $55 \%$ of the patients were operated at an age from 1 to 3 years old, and about $80 \%$ were males. It also showed that postoperatively, $60 \%$ had normal z scores, and $65 \%$ of the population still had an abnormal stool. Moreover, the study showed that $95 \%$ of the subjects returned to schooling or playing post surgery.

Conclusions Comparing it to the $96 \%$ result of Dela Merced 2003, this study had $95 \%$ of patients who were able to achieve a good functional outcome, 4-6 weeks post-surgery.

\section{IDDF2018-ABS-0063 HERBAL MEDICINE PRESCRIPTION PRACTICE FOR DYSPEPSIA IN THAI PUBLIC HOSPITALS}

${ }^{1}$ Susaraporn Sampradit*, ${ }^{2}$ Krit Pongpirul, ${ }^{3}$ Udomsak Saengow, 'Sukhamol Suksawangrote, ${ }^{3}$ Chaweewan Klongsiriwet. ${ }^{1}$ Suratthani Provincial Public Health Office, Thailand; ${ }^{2}$ Chulalongkorn University, Thailand; ${ }^{3}$ Walailak University, Thailand

\subsection{6/gutjnl-2018-IDDFabstracts.95}

Background Herbal medicine have been endorsed by policymaker as the first line treatment for common conditions. This study was aimed to comparatively assess the prescription practice of herbal and non-herbal medicine for dyspepsia in Thailand.

Methods Prescription data of 15 hospitals Surat Thani province during 2014-2016 was extracted from the electronic database by using the ICD-10 code K30. The drug names and quantity were checked for accuracy and completeness. Descriptive statistics were used for data analysis.

Results A total of 105921 dyspepsia visits by 63249 outpatients were analysed. There were 345489 drugs prescribed for dyspepsia, of which only $4.52 \%$ were herbal medicine (4.68\% in $2014,4.84 \%$ in $2015,4.11 \%$ in 2016). The top three drugs were omeprazole (21.79\%), antacid (18.51\%), and simethicone $(12.62 \%)$ whereas Curcumin capsule ranked ninth (3.54\%). For comparison, Curcumin capsule was prescribed for $3.71 \%$ in $2014,3.86 \%$ in 2015 , and $3.12 \%$ in 2016 , respectively. Most physicians prescribed only non-herbal medicine $(83.57 \%)$ while combined herbal and non-herbal drugs were prescribed in only $14.43 \%$, regardless of hospital size. Conclusions Use of Thai herbal medicine for dyspepsia slightly increased despite the national policy endorsement.

\section{\begin{tabular}{|l|l|l|l|}
\hline IDDF2018-ABS-0066 THE ADMINISTRATIVE PROJECT OF \\
\hline
\end{tabular} HELICOBACTER PYLORI INFECTION SCREENING AMONG JUNIOR HIGH SCHOOL STUDENTS IN AN AREA OF JAPAN WITH A HIGH INCIDENCE OF GASTRIC CANCER}

Chika Kusano*, Takuji Gotoda, Sho Suzuki, Hisatomo Ikehara. Division of Gastroenterology and Hepatology, Department of Medicine, Nihon University School of Medicine, Japan

\subsection{6/gutjnl-2018-IDDFabstracts.96}

Objective Helicobacter pylori infection is a common chronic infection that is closely associated with gastric cancer, known to be decreasing worldwide. We set up an administrative project of screening examination for $H$. pylori infection in junior high school students in Akita prefecture in order to investigate the current prevalence of $H$. pylori infection in childhood in an area where the incidence of gastric cancer is particularly high.

Subjects and methods All students in their second or third year of junior high school (13 to 15 year old) in two cities in Akita prefecture were recruited. First, urine-based enzymelinked immunosorbent assay and/or stool antigen tests and/or serum antibody test for detection of $H$. pylori infection was performed. Then ${ }^{13} \mathrm{C}$-urea breath test $\left({ }^{13} \mathrm{C}\right.$-UBT) was carried out in students who tested positive with one of three tests. Written informed consent was obtained from all participants and their parents.

Results A total of 2715 students were included in the $H$. pylori screening study from April 2015 to March 2017. Of these, 2619 students (96.5\%) agreed to participate and underwent a screening examination. We administered urine-based 